\title{
Retention force of zirconia bar retained implant overdenture: Clinical comparative study between PEEK and plastic clips
}

\author{
Radwa Mohsen Kamal Emera1 ${ }^{(}$, Gilan Youssef Altonbary ${ }^{1}$ \\ ${ }^{1}$ Mansoura University, Faculty of Dentistry, Department of Removable Prosthodontics, Mansoura, Egypt.
}

\section{Correspondence:}

Dr. Radwa M. K. Emera

10 Dr. Aly Basha Ebrahem, Old El Aam Hospital, Algalaa Area, Mansoura, Egypt E-mail:drradwa@yahoo.com

Received: 19 April 2019

Accepted: 19 August 2019

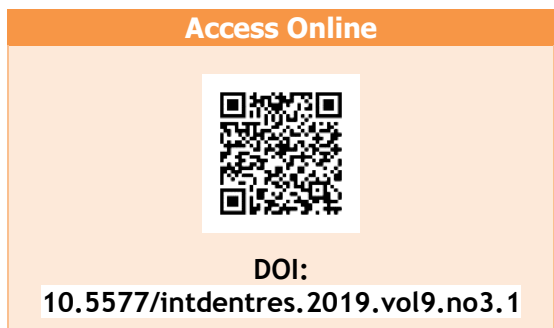

\section{Introduction}

In the implant-based prosthetic field, bar-retained dentures have become a tried and trusted treatment option for edentulous lower jaws. Up to few years ago, non-precious-metal alloys and titanium were considered the materials of choice for bar construction. However, metal-free prostheses are now gaining a

\begin{abstract}
Aim: This study aimed to evaluate and compare retention force of two implants retained mandibular overdenture with zirconia bar using two different clip materials.

Methodology: 20 completely edentulous patients $(8$ women and 12 men) with age ranged between 45 and 65 years were selected for this study. All patients received conventional maxillary and mandibular complete dentures. Two implants were inserted bilaterally in the mandibular canine region. CAD-CAM fabricated zirconia bar attachment was used to retain mandibular overdentures. Patients were randomly divided into two equal groups where poly-oxy-methylene (POM) clip was used for Group I and poly-ether-ether-ketone (PEEK) clip for Group II. Retention force was measured at time of overdenture insertion (T0), six months (T6), and twelve months (T12) later.

Results: Significant difference was recorded between the two groups in initial retention forces (TO) where PEEK clip group recorded higher retention forces in comparison to POM clip group $(P=0.029)$. However, insignificant difference was observed at (T6) and (T12). Within group comparison of mean retention values at different follow-up periods revealed significant difference for both groups.

Conclusions: Within parameters of this study, it could be concluded that both POM and PEEK retentive clips can provide acceptable and comparable retention forces when used with zirconia bar during 12 months period of overdenture use.
\end{abstract}

Keywords: Zirconia bar, POM clip, PEEK clip, implant overdenture.

How to cite this article: Emera RMK, Altonbary GY. Retention force of zirconia bar retained implant overdenture: Clinical comparative study between PEEK and plastic clips. Int Dent Res 2019;9(3):92-8. great importance supported by the high-precision CAD/CAM manufacturing process that enables the use of biocompatible and aesthetic materials. Zirconium oxide (zirconia) has become a promising material for fabricating bar attachment due to its high strength, biocompatibility, and realistic color (1). In addition, zirconia bar can be easily constructed using CAD/CAM technology with elimination of many technical steps 
and errors involved in the conventional casting procedures (2).

Materials used for fabrication of attachment systems should be wear resistant to maintain stable retention force overtime (3). Wear of retentive clips concerning bar-clip attachments was documented to adversely affect retention (4). Less changes in retention force were recorded with plastic retentive clips made of poly-oxy-methylene (POM) in comparison to metal ones, mainly due to their higher resiliency and proper modulus of elasticity, therefore the plastic clips became more widely used $(5,6)$.

Recently, poly ether ether ketone (PEEK) material was documented to possess high strength, insolubility in common solvents, wear resistance and excellent biocompatibility (7-9). Thus, this material was introduced as retentive clip matrices $(3,10)$. Bayer et al, compared the clinical performance of PEEK retentive clips with those made of poly-oxy-methylene (POM) as the standard clip material and concluded that both materials showed satisfying results as a retentive clip with round metal bars (3).

Hammas et al, evaluated the retention force of mandibular implant retained overdentures using metal and PEEK bar attachments with either POM or PEEK clips. The results suggested that the invitro performance of PEEK clips was superior to those made of POM, where the highest mean values of retention were found in (PEEK bar and PEEK clip group) followed by (Metal bar and PEEK clip group) and (PEEK bar and POM clip group) while the least mean values of retention were found in (Metal bar and POM clip group) (10).

By reviewing the literature, very limited data is available about the retention force of different clip materials with zirconia bar. Consequently, the aim of this clinical study was to evaluate and compare the retention force of two implants retained mandibular overdenture with zirconia bar using either conventional plastic clips or PEEK ones after a period of 12 months. Null hypothesis was that the change in retentive force would not differ among the different clip materials.

\section{Materials and Methods}

A total of 20 completely edentulous patients (8 women and 12 men) with age ranged between 45 and 65 years were selected from the clinic of the Removable Prosthodontics Department, Faculty of Dentistry, Mansoura University. The patients had a sufficient restorative space (at least $15 \mathrm{~mm}$ ) to allow space for bar construction. Adequate bone quantity and quality in the mandibular canine area bilaterally was verified by preoperative cone-beam $\mathrm{CT}$ to receive standard implants of $15 \mathrm{~mm}$ length and $3.6 \mathrm{~mm}$ diameter.

Patients with history of parafunction habit, bone metabolic disorders (as uncontrolled diabetes and hyper- parathyroidism), or history of radiotherapy to the head and neck region were excluded.
Approval of this study protocol was done by the Faculty Ethical Committee. All patients signed written consents after informing them about the detailed line of treatment and the needed follow up visits.

\section{Surgical and prosthetic procedures:}

Conventional maxillary and mandibular dentures were constructed for all patients where artificial acrylic teeth were arranged following the lingualized balanced occlusal concept. Two dental implants (15

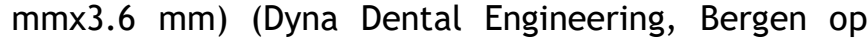
Zoom, the Netherlands) were inserted in the mandibular canine region bilaterally using standardized 2-stage submerged surgical protocol. Refitting of mandibular dentures to the residual ridges was done using a soft relining material (Ufi Gel, Voco, Cuxhaven, Germany), followed by refining of occlusion.

After three months, the implants were uncovered using tissue punch and healing abutments were connected to them. Mandibular acrylic resin custom trays were fabricated with an opening corresponding to implants sites. Long impression transfer copings were screwed to the fixtures and splinted by Duralay acrylic resin (Inlay pattern resin. Dental Mfg. Co. USA). An open-tray impression was made using medium body polyether impression material (3MTM Impregum TM). The impression was poured in dental stone after connecting the implant analogs to the impression copings.

\section{CAD/CAM-manufacturing of zirconium oxide bar:}

Two Ti base abutments (Dyna Dental Engineering, Bergen op Zoom, the Netherlands) were screwed to the implant analogs on the model. Two scan bodies (panadent.co.uk) were connected to the $\mathrm{Ti}$ base abutments then the model was scanned with the 3shape D800 lab scanner (3Shape 3D Dental Scanners, Wieland dental) (Fig. 1). The collected data were imported to the CAD software for bar designing (CAD/CAM, 3 Shape Dental System ${ }^{\text {TM }}$ ). Hader bar design was selected from the software library and applied with preservation of $1 \mathrm{~mm}$ supragingival hygienic space (Fig. 2).

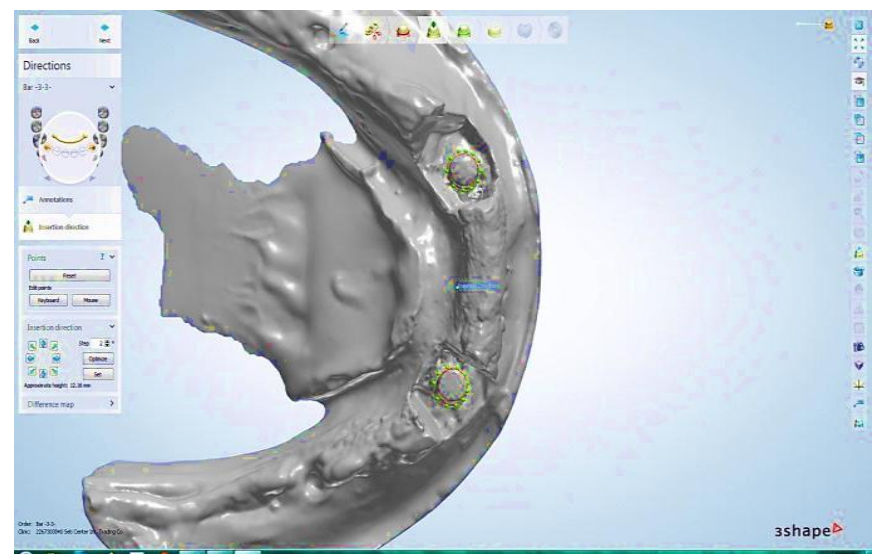

Figure 1. Three dimensional image of the scanned model including the two implant abutments. 
The CAD data was sent to the milling machine (Wieland dental, ivoclar vivodent, Zenotic select hybrid) to mill the bar assembly, from semi-sintered zirconia blanks (Zenostar MO 2, Wieland dental, ivoclar vivodent). After sintering of the bar, it was fitted to the Ti base abutments precisely on the model (Fig. 3). Duplication was done to the model while the bar was secured in its place.

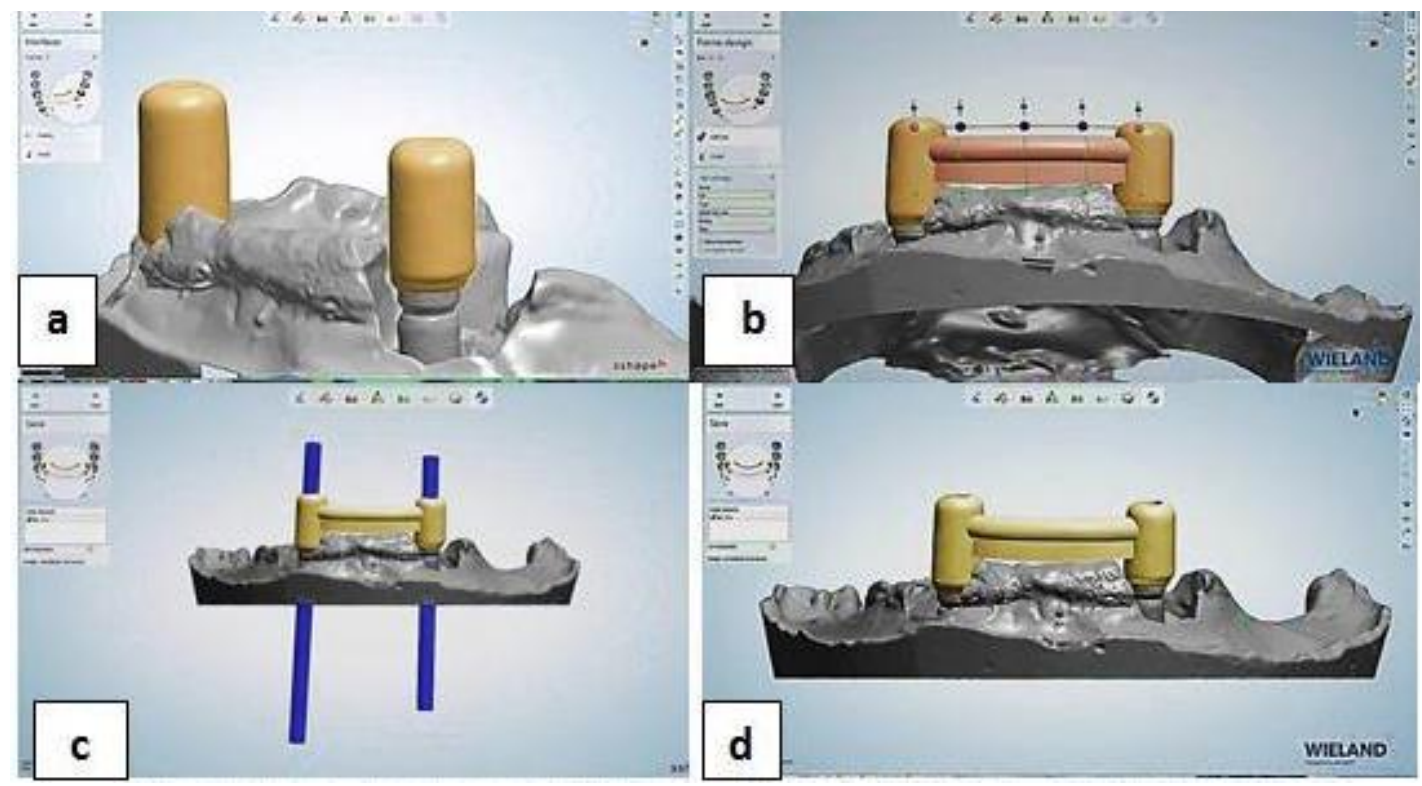

Figure 2. Designing of zirconia bar: (a). Software design of zirconia bar abutments. (b) Applied Hader bar software design. (c) Path of insertion represented by blue lines. (d) Final software design of bar assembly.

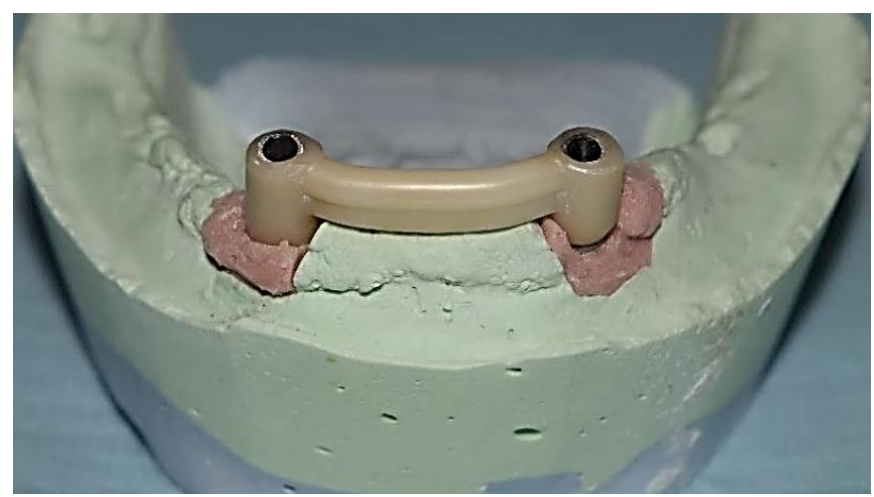

Figure 3. Finished zirconia bar assembly.

\section{Mandibular Overdenture construction:}

Duplication of the mandibular denture polished and occlusal surfaces was done using a silicone index (Coltoflax; Coltene AG, Altstatten, Switzerland). Identical acrylic resin teeth were positioned in their respective places in the silicone index. The index was repositioned against the duplicate mandibular stone cast. The mold cavity was filled with molten base plate wax that was flasked to fabricate a duplicate denture with similar bulk and contour.

\section{Patients grouping:}

The patients were randomly assigned into two equal groups using balanced randomization computer program, with respect to patient's sex and age. Assigning was done according to material of the retentive clip used; POM (Yellow, Medium retention, RHEIN 83. Italy) was used for group I and milled PEEK clip (JUVORA, Invibio/ JUVORA Ltd. UK. Unfilled PEEK) that is identical to the scanned POM clip used for group II (Fig. 4).

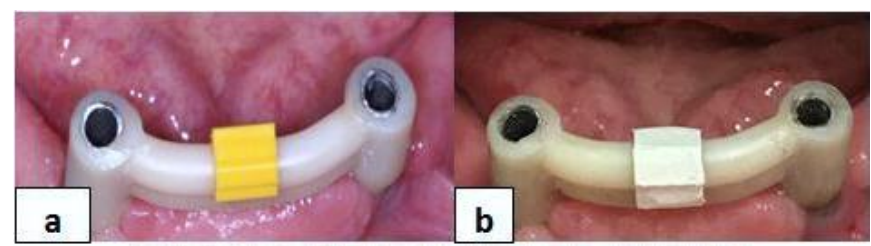

Figure 4. (a)- Plastic retentive clip of group I. (b) PEEK retentive clip of group II.

Ti base abutments were secured to the implants and the bar assembly was cemented to them with selfadhesive resin cement (seT, SDI, Australia). Clips were picked up in the patient mouth with auto-polymerized acrylic resin while the patient was lightly biting in centric occlusion (Fig. 5). Final refining of occlusion was done.

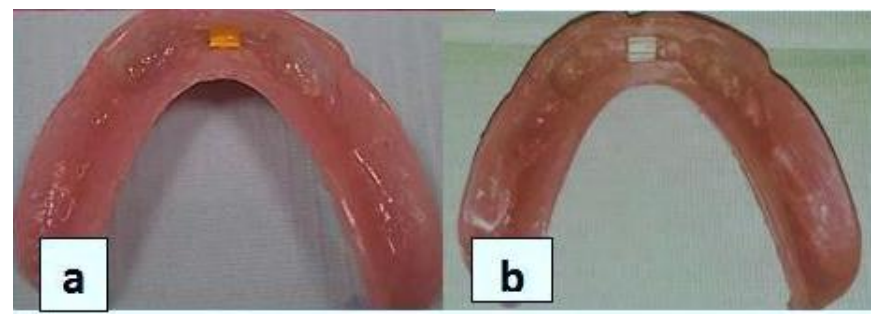

Figure 5. Overdenture fitting surface showing Picked up: (a) plastic retentive clip, (b) PEEK retentive clip.

\section{Evaluation of retention force:}

Retention force was evaluated at the time of overdenture insertion (T0), six months (T6) and twelve months (T12) later by measuring maximum dislodging 
force to separate the overdenture from the attachment. Evaluation was done using the device developed by Hussein and Elsyad (11) through the following steps:

- Four right angle metal hooks were attached to the overdenture at the same horizontal high at canine and first molar areas bilaterally (Fig. 6). - The patient was instructed to wear the mandibular overdenture and remove the opposing maxillary denture followed by placing his chin at the chin rest of the device while allowing the fork to be under the four hooks (Fig. 7).

- The wheel of the device was rotated to move the forcemeter vertically until the overdenture was removed from its place. The forcemeter reading was recorded. This step was repeated three times and the mean was calculated.

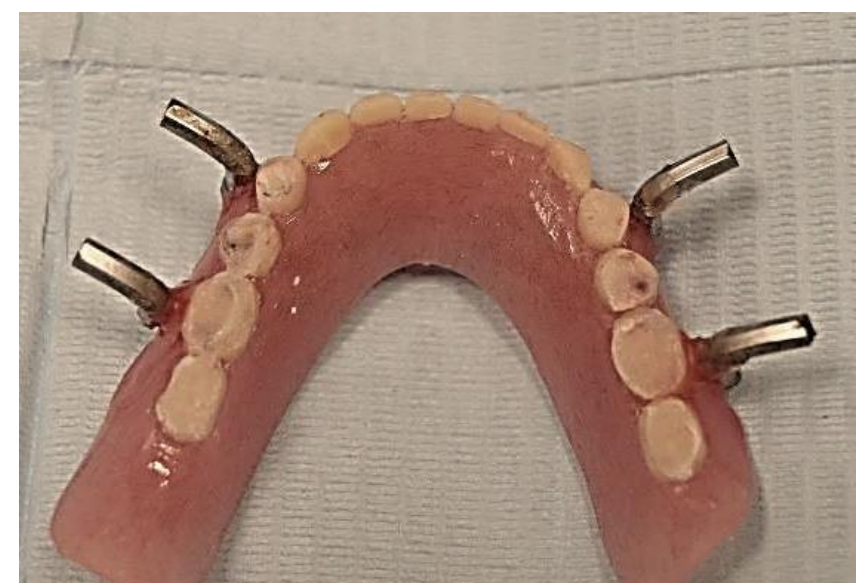

Figure 6 . Four right angle metal hooks were attached to the overdenture.

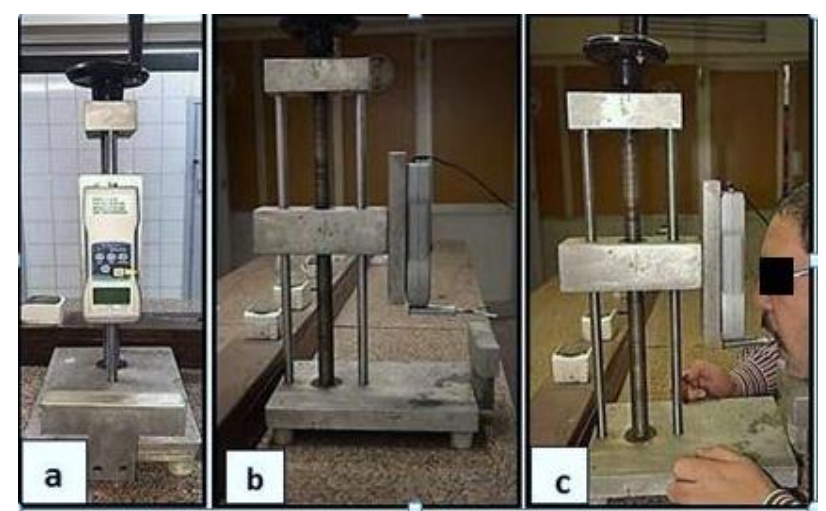

Figure 7. Evaluation of retention force: (a) Frontal view of the device showing the forcemeter instrument. (b) Lateral view of the device showing the fork and the chin rest. (c) The patient placing his chin at the chin rest of the device while allowing the fork to be under the four hooks.

\section{Statistical Analysis}

Obtained data was analyzed by SPSS computer software (Version 21 SPSS, Chicago. IL, USA). Normal distribution of data was evaluated using one sample Kolmogorov Smirnov test. Normally distributed continuous data were described as (mean \pm standard deviation) unpaired sample t-test was applied to compare the two groups. Multivariate analysis (general linear model) was applied for comparison of all observational periods within each group, and paired sample t-test was applied for comparison of each two periods. ( $P$ ) was significant if $<0.05$ at $95 \%$ confidence interval.

\section{Results}

Mean retention values at time of overdenture insertion (T0), after six months (T6) and after twelve months (T12) of overdenture use of both groups are presented in (Table 1 and Fig. 8) Comparisons between the two groups showed significant difference in initial retention forces (TO) where PEEK clip group recorded higher retention forces in comparison to POM clip group $(\mathrm{P}=0.029)$. However, insignificant difference was observed at T6 and T12. Within group comparison of mean retention values at different follow-up periods revealed significant difference for both groups.

Comparison of retention values between each two follow-up periods within each group, revealed significant differences except between (T6 - T12) for PEEK clip group $(P=0.228)$ and $(T 0-T 6)$ for POM clip group $(P=0.072)$ as shown in (Table 2$)$.

Table 1. Showing mean values of retention of both groups at different follow-up periods

\begin{tabular}{|c|c|c|c|}
\hline & $\begin{array}{c}\text { PEEK } \\
(M \pm S D)\end{array}$ & $\begin{array}{c}P O M \\
(M \pm S D)\end{array}$ & $\begin{array}{l}\text { Unpaired sample t-test } \\
\text { (P value) }\end{array}$ \\
\hline TO & $11.17 \pm 1.28$ & $8.46 \pm 0.83$ & $0.029^{*}$ \\
\hline T6 & $8.28 \pm 0.37$ & $7.50 \pm 0.54$ & 0.394 \\
\hline T12 & $7.93 \pm 0.55$ & $6.27 \pm 0.61$ & 0.112 \\
\hline $\begin{array}{l}\text { Multivariate analysis } \\
\quad(p \text { value })\end{array}$ & $0.005^{*}$ & $0.011^{*}$ & \\
\hline
\end{tabular}

M: mean SD: standard deviation

*: significant $(P<0.05)$

T6: after 6 months of overdenture use
T0: at time of overdenture insertion

T12: after 12 months of overdenture use 


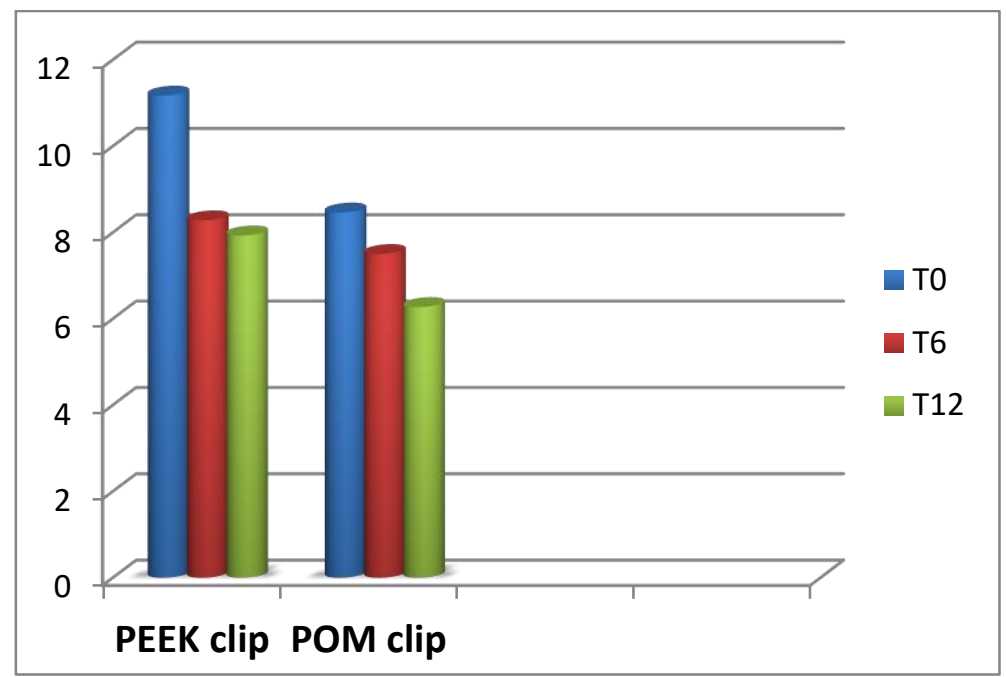

Graphic 1. Showing mean values of retention of both groups at different follow-up periods

Table 2. Showing within group comparison of retention values between each two follow-up periods

\begin{tabular}{clc|} 
& PEEK & POM \\
\hline & $\begin{array}{c}\text { Paired sample t-test } \\
(\mathrm{P} \text { value }) \\
\text { Peek }\end{array}$ & POM \\
\hline T0-T6 & $0.000^{*}$ & 0.072 \\
T6-T12 & 0.228 & $0.024^{*}$ \\
T0-T12 & $0.006^{*}$ & $0.007^{*}$ \\
\hline *: significant $(\mathrm{P}<0.05)$ & T0: at time of overdenture insertion & \\
T6: after 6 months of overdenture use & T12: after 12 months of overdenture use
\end{tabular}

\section{Discussion}

Zirconium oxide material was previously applied in milled bar construction with two distal additional retaining elements and peek superstructure $(1,12)$ or with a corresponding zirconia complete overdenture (13). However, this research introduced the application of zirconia for Hader bar construction that retain a heat-cured acrylic resin mandibular overdenture with either POM or PEEK clips. The hader bar design was selected for the two implants retained overdenture used in this study to permit hinging movement and allow occlusal load distribution between implants and posterior residual ridges.

Results of invitro studies that evaluated retention forces of different attachment systems cannot be applied on the clinical situation. microscopical examination of different attachments revealed that POM or nylon components were deformed during fatigue tests of invitro studies, which may increase values of retention force. Another suggested cause for this retention increase is the POM thermal expansion during the test. However, under clinical conditions, saliva composition, oral environment, and temperature may influence the results (14). Masticatory and parafunction forces also cannot be simulated in invitro studies. Therefore, this study was concerned with the clinical evaluation and comparison of POM retentive clips and the recently suggested PEEK ones.

The measuring protocol followed in the study of Williams et al, allowed the identification of whether wear and the subsequent decrease in retention occurred at the matrix or patrix level. The results indicated that the plastic retentive clips and not the round bars were responsible for the retention loss (15). This is in consistence with the clinical experience that the exchange or activation of the matrix can easily compensate loss of retention (16). These finding explain the importance of testing clinical performance of different clip materials especially when used with a recent bar material as zirconia.

Retention force was measured by evaluating peak loads or maximum dislodging forces, that is defined as the maximum developed forces till complete separation of attachment components from teeth or 
implant abutments, and commonly used as the measurement of prosthesis retention (6).

The results of the present study showed significant difference in values of initial retention forces (T0) where PEEK clip group recorded higher retention forces in comparison to POM clip group. However, insignificant difference was observed at (T6) and (T12) where the null hypothesis could not be rejected except for (T0). This result is in accordance to the study of Bayer et al, that reported no statistically significant difference in the clinical performance of the retentive clips made of PEEK or POM with metal bar (3). Both clip materials showed a decrease in retention force with no significant difference between them after 6 months of overdenture use. Moreover, mean values of retention force observed in the present study for PEEK retentive clips $(7.93 \mathrm{~N})$ and POM ones $(6.27 \mathrm{~N})$ with zirconia bar are comparable to those observed with metal bar where PEEK and POM clips recorded $(7.85 \mathrm{~N})$ and $(7.44 \mathrm{~N})$ respectively after 6 months of overdenture use.

Within group comparison of mean retention values at different follow-up periods revealed significant difference for both groups. Significant difference was observed between (T0) and (T6) for PEEK clip group while retention loss was insignificant in the next six months in contrast to the POM clip group. Bayer et al, concluded that retention forces with peek clips decreased during the first three months of the overdenture in function and stabilized afterwards (3).

Hammas et al, concluded that the advantage of PEEK retentive clip compared to POM material could be demonstrated during the simulation period of 12 months of overdenture use. Both materials showed in vitro acceptable results toward retention force however, PEEK showed more resistance to wear than POM material with metal or PEEK bars (10).

The type of PEEK material used in this study was the unfilled one, the issue that may explain the significant loss of retention especially in the first three months of overdenture use. It was documented that performance of PEEK composites in tribological tests is better than that of pure PEEK (17). Micro and nanosized filler in PEEK was suggested to provide lower friction coefficients and less wear rate than the unfilled type $(18,19)$.

\section{Conclusions}

Within parameters of this study, it could be concluded that both POM and PEEK retentive clips can provide acceptable and comparable retention forces when used with zirconia bar during 12 months period of overdenture use.

\section{Recommendations:}

Further studies are indicated to evaluate:

1- Long-term clinical performance of PEEK retentive clips compared to those made of POM.

2- Clinical performance of retentive clips made of different filled PEEK materials with zirconia bar.
Ethical Approval: Ethics committee approval was received for this study from Mansoura University.

Peer-review: Externally peer-reviewed.

Author Contributions: Conception R.M.K.E.; Design - R.M.K.E., G.Y.A. Supervision-R.M.K.E.; Materials - G.Y.A.; Data Collection and/or ProcessingR.M.K.E., G.Y.A.; Analysis and/or Interpretation R.M.K.E., G.Y.A.; Literature Review - R.M.K.E., G.Y.A.; Writer - R.M.K.E.; Critical Review R.M.K.E., G.Y.A.

Conflict of Interest: Noconflict of interest was declared by the authors.

Financial Disclosure: The authors declared that this study has received no financial support.

\section{References}

1. Freitag D. Case Report-A tried and trusted concept in a new formula. Funktionelle und ästhetische Zahntechnik 2014;6:1922.

2. Goo C, Tan K. Fabricating CAD/CAM implant-retained mandibular bar overdentures: A clinical and technical overview. Case Rep Dent 2017;2017:id 9373818. (Crossref)

3. Bayer S, Komor N, Kramer A, Albrecht D, Mericske-Stern R, Enkling N: Retention force of plastic clips on implant bars: a randomized controlled trial. Clin. Oral Impl. Res 2012;23:137784. (Crossref)

4. Van Kampen F, Cune M, Van Der Bilt A, Bosman F. Retention and post insertion maintenance of bar- clip, ball and magnet attachments in mandibular implant overdenture treatment: an in vivo comparison after 3 months of function. Clin. Oral Impl. Res 2003;14(6):720-6. (Crossref)

5. Bayer S, Gruner M, Keilig L, Hultenschmidt R, Nicolay C, Bourauel C, Utz KH. Investigation of the wear of prefabricated attachments - an in vitro study of retention forces and fitting tolerances. Quintessence Int 2007;38:229-37.

6. Fromentin $\mathrm{O}$, Lassauzay $\mathrm{C}$, Abi Nader $\mathrm{S}$, Feine $\mathrm{J}$, de Albuquerque Junior RF. Testing the retention of attachments for implant overdentures - validation of an original force measurement system. J Oral Rehabil. 2010;37:54-62. (Crossref)

7. Liebermann A, Wimmer $T$, Schmidlin $P R$, Scherer $H$, Löffler $P$, Roos M, Stawarczyk B. Physicomechanical characterization of polyetheretherketone and current esthetic dental CAD/CAM polymers after aging in different storage media. J. Prosthet. Den 2016;3:321-8. (Crossref)

8. Tekin S, Cangül S, Adıgüzel Ö, Değer Y. Areas for use of PEEK material in dentistry. Int Dent Res 2018;8(2):84-92. (Crossref)

9. Tekin S, Adıgüzel Ö, Cangül $\mathrm{S}$. An evaluation using micro-CT data of the stress formed in the crown and periodontal tissues from the use of PEEK post and PEEK crown: A 3D finite element analysis study. Int Dent Res 2018;8(3):144-50. (Crossref)

10. Hammas M, El-Saadawy M, El-Agamy A. Effect of Different Bar Attachment and Clip Materials on Retention Force for Mandibular Implant Supported Overdentures (An Invitro Study). ADJ-for Girls 2018; 5(2):195-204. (Crossref)

11. Hussein $M$, Elsyad $M$. A new device measures retention clinically for implant retained overdenture. EDJ 2017;63: 1521-4.

12. Spies B, Petsch M, Kohal R, Beuer F. Digital Production of a Zirconia, Implant-Supported Removable Prosthesis with an Individual Bar Attachment Milled from Polyether Ether Ketone: A Case History Report. Int J of Prosthodontics 2018;31(5):4714. (Crossref) 
13. Bühler NM, Teubner E, Marinello CP. Zirconia in removable prosthodontics - A case report (in German). Schweiz Monatsschr Zahnmed 2011;12:659-68.

14. Botega DM, Mesquita MF, Henriques GE, Vaz LG. Retention force and fatigue strength of overdenture attachment systems. J Oral Rehabil 2004;31(9):884-9. (Crossref)

15. Williams BH, Ochiai KT, Baba T, Caputo AA: Retention and load transfer characteristics of implant-retained auricular prostheses. Int J oral maxillofac Impl 2007;22:366-72.

16. Zitzmann NU. A review of clinical and technical considerations for fixed and removable implant prostheses in the edentulous mandible. Int J of Prosthodontics 2002;15:65-72.
17. Lind J, Lindholm P, Qin J, Kassman Rudolphi A. Friction and wear studies of some PEEK materials. Finnish $\mathrm{J}$ of Tribology 2015;33(2):20-28.

18. Shao X, Xue Q. Effect of Nanometer and Micrometer $\mathrm{SiO}(2)$ Particles on the Tribology Properties of Poly (phthalazine ether sulfone ketone) Composites. Materials and Mechanical Engineering 2004;28(6):39-42

19. Skirbutis G, Dzingutè A, Masiliūnaitè V, Šulcaitè G, Žilinskas J. $A$ review of PEEK polymer's properties and its use in prosthodontics Stomatologija, Baltic Dental and Maxillofacial Journal 2017;19:19-23. 